\title{
Unicellular-multicellular evolutionary branching driven by resource limitations
}

\author{
Adriano Bonforti ${ }^{1,2}$ and Ricard Solé*1,2,3 \\ ${ }^{1}$ ICREA-Complex Systems Lab, UPF-PRBB, Dr. Aiguader 80, 08003 Barcelona \\ ${ }^{2}$ Institut de Biologia Evolutiva, CSIC-UPF, Passeig Maritim de la Barceloneta 37, 08003 Barcelonacritical \\ ${ }^{3}$ Santa Fe Institute, 1399 Hyde Park Road, Santa Fe NM 87501, USA.
}

\begin{abstract}
Multicellular life forms have evolved many times in our planet, suggesting that this is a common evolutionary innovation. Multiple advantages have been proposed for multicellularity (MC) to emerge. In this paper we address the problem of how the first precondition for multicellularity, namely "stay together" might have occurred under spatially limited resources exploited by a population of unicellular agents. Using a minimal model of evolved cell-cell adhesion among growing and dividing cells that exploit a localised resource with a given size, we show that a transition occurs at a critical resource size separating a phase of evolved multicellular aggregates from a phase where unicellularity (UC) is favoured. The two phases are separated by an intermediate domain where where both UC and MC can be selected by evolution. This model provides a minimal approach to the early stages that were required to transition from Darwinian individuality to cohesive groups of cells associated with a physical cooperative effect: when resources are present only in a localised portion of the habitat, MC is a desirable property as it helps cells to keep close to the available local nutrients.
\end{abstract}

Keywords: Evolutionary transitions, multicellularity, cell adhesion, evolutionary preconditions, statistical physics

\section{INTRODUCTION}

Being multicellular implies moving away from the pure Darwinian replicator associated with single-cell life forms. It necessarily requires more resources to maintain a population of cells and needs expanding the molecular toolkit by exploiting (or adding) new cell-cell interaction mechanisms. Why evolve such complexity when unicellular (UC) agents can do well and have a much smaller cost? The fact that multicellularity (MC) has evolved in our biosphere many times suggests that the $\mathrm{UC} \rightarrow \mathrm{MC}$ transition is a favourable event under a wide range of circumstances (Knoll, 2011; Ruiz-Trillo et al., 2015).

Multicellularity might have arisen in the first place for a number of different reasons. The common thread is usually the presence of some kind of cooperative effect. As pointed out in (Bonner, 2009), the fact that has evolved so many times strongly suggests that "there has been a significant selection for it in the ancient cellular world". Indeed, we can identify a diverse range of selective drivers that might have operated in the first steps towards multicellularity, but also shown to be in action in artificially evolved multicellular aggregates. They include mechanisms of predator avoidance (associated to larger assemblies), faster sedimentation, stress resistance or division of labor, among others (Tong et al., 2021). The selective pressure is here associated to a specific, efficient functional trait.

In this paper we adopt a different approximation by asking about the preconditions that immediately precede the landscape of simple multicellularity. Knoll defines

\footnotetext{
*Corresponding author. email: ricard.sole@upf.edu
}

MC organisms as the group including "filaments, clusters, balls, or sheets of cells that arise via mitotic division from a single progenitor" (Knoll, 2011). In this context, a MC agent is not only a coherent association of cells: it is generated through a process of development of a life cycle where groups, instead of individuals, are the new self-replicating entities. However, a first step towards group replication requires the formation of the group as a necessary precondition and comes at a cost to free-living fitness (Queller 2000; Ratcliff et al., 2012; Libby and Ratcliff, 2014).

The approach taken here is inspired by three main concepts. The first is that, as it occurs with the transition to eusociality, where a crucial preadaptation is the fidelity of agents to their nests, a requirement for multicellular groups is the presence of cell-cell interactions that guarantee the formation of cohesive groups of cells (Tarnita et al., 2013; Biernaskie and West, 2015; Pentz et al. 2020). As will be discussed here, advantages related to group performance among essentially identical UC agents can evolve before the emergence of division of labour under environmental stress (see Duran-Nebreda et al., 2016).

Secondly, a proper approximation requires to consider embodied agents: MC is grounded in a physical interaction among discrete cells that occurs in a given spatial embedding. Cell-cell attachment is actually a crucial part of the generic physical mechanisms that pervade multicellular programs (Newman and Comper, 1990; Newman and Baht, 2008; Newman et al., 2006). Here cell adhesion is a physical interaction that has a cost and plays a key role in the generation of developmental complexity and has been shown to explain several classical experiments of regeneration (Forgacs and Newman, 2005). Finally, MC systems emerge in an ecological context where resources are relevant and their scarcity or spatial limitation au- 
b

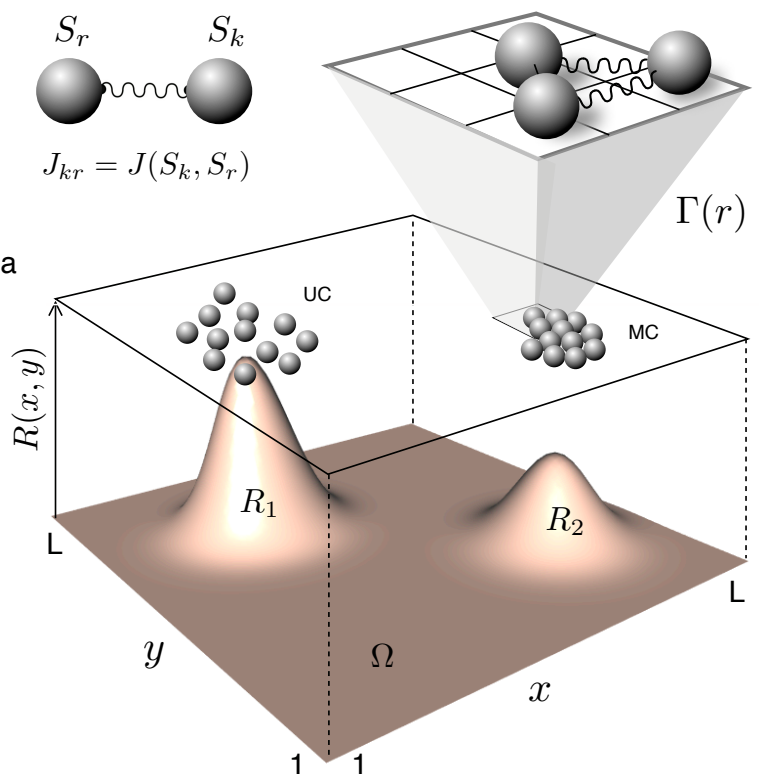

FIG. 1: Spatial dynamics of in silico cell units on the $\Omega$ lattice with a continuous diffusion of resources. Different resource abundances $\left(R_{1}\right.$ and $\left.R_{2}\right)$ are used to mimic diverse levels of local resources. These different resource sizes favour the evolution of two main types of behaviour, namely unicellular (UC) and multicellular (MC). (b) pairwise interactions are weighted by a coupling constant $J_{k r}$ that averages the adhesion constants of the pair. Interactions occur among nearest neighbours (c) on the $3 \times 3$ neighbourhood.

tomatically impose selection pressures to the embodied $\mathrm{UC}$ agents. What is the impact of resource limitations as a trigger to evolve MC clusters of cells? What kind of primitive cooperation might emerge due to a purely physical association among single cells?

Here we explicitly introduce population growth, cell adhesion and resource limitation in a toy model of evolved MC. A given amount of nutrient, localised in space, is exploited by a population of cells that consume it to grow and reproduce while evolving adhesion. Since moving away from local resources can be detrimental to single cells, evolution towards adhesive interactions is favoured when resources are available only in a reduced portion of the habitat, thus showing that in presence of localised resources a simple embodied description of a cell population can develop a cooperative effect that allows MC to appear.

In this paper we describe the model assumptions as well as the conditions under which both UC and MC evolve as two different phases of our model, separated by a marked phase transition between them.

\section{METHODS}

In our in-silico model cell populations evolve on a twodimensional $L \times L$ square lattice $\Omega$ (fig. 1a),

$$
\Omega=\{r=(i, j) \mid 1 \leq i, j \leq L\}
$$

where both cells and a resource field will coexist. Here the state of each site $r=(i, j)$ is defined by three variables. One is the state $S_{r} \in \Sigma$ where the set $\Sigma=\{0,1\}$ includes empty $\left(S_{r}=0\right)$ and occupied $\left(S_{r}=1\right)$ sites. Additionally, each cell carries a given adhesion parameter $J_{r}$ that will be required to determine its affinity with other nearest cells in a local neighbourhood $\Gamma_{r}$ (fig. 1b). In our model cells can have a negative or positive adhesion parameter, which favours adhesion or active repulsion with other cells, respectively. By active repulsion we mean that cells with a positive adhesion parameter will actively try to move away from other cells. The interaction of a cell with medium is neutral (i.e. equal to zero). Finally, cells have an associated biomass (or internal energy) $B(r)$.

The energy contribution of a given state $S_{r}$ to the system is here defined as the sum of the pairwise interaction of the state with each one of its neighbours:

$$
\mathscr{H}_{r}=\sum_{S_{\mu} \in \Gamma_{r}}\left(\frac{J_{r}+J_{\mu}}{2}\right) S_{r} S_{\mu}
$$

where the effective interaction between $S_{r}$ and $S_{\mu}$ (Fig. $1 b)$ is provided by the average $\left(J_{r}+J_{\mu}\right) / 2$ and the product $S_{r} S_{\mu}$ takes in account the fact that interaction of cells with empty sites is equal to zero. Due to our definition of $J$, the smaller the values of the adhesion constants, the lower the energy of the system will be.

Adhesion dynamics occurs by means of an energy minimisation process (Steinberg, 1975, 1985; Foty and Steinberg 2005; Deutsch and Dormann, 2005). Cells can move around and are able to swap their positions with neighbouring cells. The swap is more probable to occur if it minimises the energy of the system. The overall result is that the stronger the adhesion value, the more cells will stay together and local movement will be limited. Each step in the dynamics involves choosing a random site $S_{r}=1 \in \Omega$ (i.e. a site occupied by a cell). We then choose a neighbour $S_{r^{\prime}}$ (which can be occupied or empty). We calculate the initial energy of the system $\mathscr{H}$ and also the energy $\mathscr{H}^{\prime}$ that would result from swapping $S_{r}$ by $S_{r^{\prime}}$. The calculation is limited to the neighbourhoods of these two sites, and thus the initial energy is given by the sum of the interactions of $S_{r}$ and $S_{r^{\prime}}$ with their respective neighbours:

$$
\mathscr{H}=\sum_{S_{\mu} \in \Gamma_{r}}\left(\frac{J_{r}+J_{\mu}}{2}\right) S_{r} S_{\mu}+\sum_{S_{\nu} \in \Gamma_{r^{\prime}}}\left(\frac{J_{r^{\prime}}+J_{\nu}}{2}\right) S_{r^{\prime}} S_{\nu}
$$

An equivalent expression is then obtained for the energy of the system after swapping $\left(\mathscr{H}^{\prime}\right)$. 
TABLE I: Model parameters

\begin{tabular}{lll}
\hline Name & Description & Value \\
\hline $\mathrm{T}$ & Temperature (noise) parameter & 1 \\
$\rho_{R}$ & Resource input rate per site & 0.085 \\
$\eta_{R}$ & Resource natural degradation & 0.005 \\
$\xi_{R}$ & Resource absorption by cells & 0.001 \\
$D_{R}$ & Resource diffusion factor & 0.05 \\
$q$ & Number of neighbors & 8 \\
$\eta_{B}$ & Cell's internal energy degradation & 0.1 \\
$B_{\min }$ & Minimum allowed biomass & 0.01 \\
$B_{\max }$ & Maximum allowed biomass & 0.05 \\
$P_{\mu}$ & Probability of mutation & 0.005 \\
$\sigma_{\mu}$ & Mutation distribution sigma & 0.005 \\
$\Delta J$ & Maximum change in adhesion $J$ & 0.1 \\
\hline
\end{tabular}

The two sites $S_{r}$ and $S_{r^{\prime}}$ will switch their positions with a higher probability if the configuration with swapped positions is energetically more favorable than the original configuration, i.e. if the $\Delta \mathscr{H}=\mathscr{H}^{\prime}-\mathscr{H}<0$. The probability of swapping $P\left(S_{r} \leftrightarrow S_{k}\right)=P(k \mid r)$ follows a Boltzmann distribution:

$$
P(k \mid r)=\frac{1}{1+e^{\Delta H / T}}
$$

so that the probability of swapping is higher for $\Delta \mathscr{H}<0$ and becomes smaller as $\Delta \mathscr{H}$ increases. The parameter $T$ represents the temperature of the system (noise term), so that for $T$ large enough we have $P\left(S_{r} \leftrightarrow S_{k}\right) \approx 1 / 2$ (i.e. the swapping will occur randomly). For a single cell in empty space we also have $P\left(S_{r} \leftrightarrow S_{k}\right)=1 / 2$, since $\Delta \mathscr{H}=0$.

The second part of the model deals with the resource field $R(r)$ (Fig. 1a). An external nutrient is introduced at a rate $\rho_{R}$ within a circular area $\Psi \in \Omega$ of diameter $\phi$. Decay, consumption y cells and diffusion occur, following:

$$
\frac{\partial R_{r}}{\partial t}=\rho_{R} \delta_{(r \in \Psi)}-\left(\eta_{R}-\xi_{R} S_{r}+D_{R} \nabla^{2}\right) R(r)
$$

Where the last term in the rhs introduces the diffusion term, to be approximated by:

$$
\nabla^{2} R(r)=\left(\sum_{k \in \Gamma_{r}} R(k)-q R(r)\right),
$$

where the term $\delta_{r \in \Psi}$ is 1 if $r \in \Psi$ and zero otherwise.

Cells vary their biomass (internal energy) according to the following equation:

$$
\frac{\partial B_{r}}{\partial t}=\left(\xi_{R} R_{r}-\eta_{B} B_{r}\right) S_{r}
$$

And will grow as a result of nutrient absorption: their biomass will increase as $B_{r} \rightarrow B_{r}+\xi_{R} R_{r}$. However, there is a cost to maintain the living state, with a metabolic cost introduced as a decay $B_{r} \rightarrow B_{r}\left(1-\eta_{B}\right)$. a
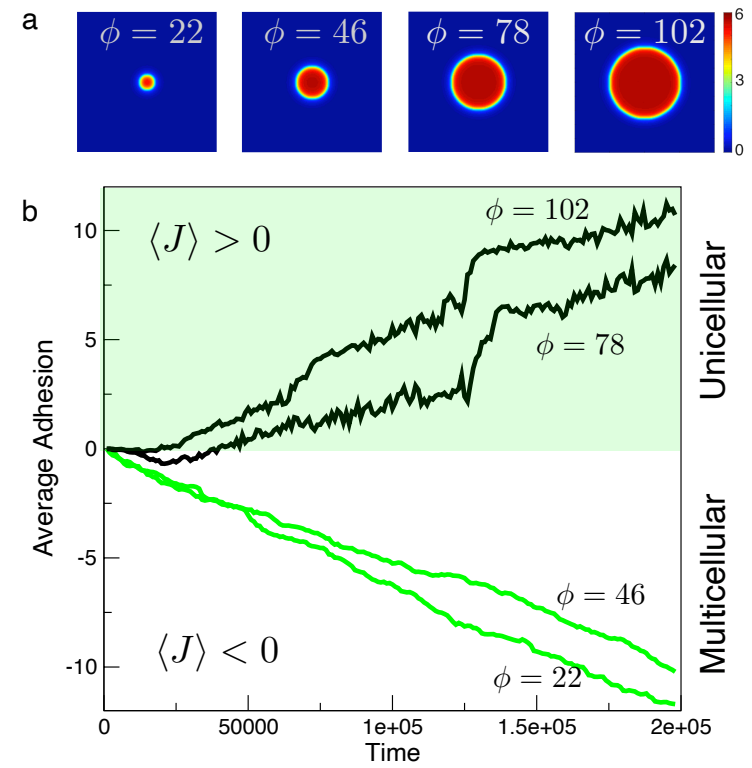

FIG. 2: Evolution of adhesion (population-level average) for different sizes of the central nutrient disc (color bar scale is in $\times 10^{-2}$ a. u. $)$. For small resource sizes $\left(\phi<\phi_{c}\right)$ the $J$ parameter adhesion evolves towards adhesion $(J<0)$, whereas for larger resources unicellularity (repulsion) is selected $(J>0)$. On top we show the corresponding resource spatial snapshots associated with each of the four evolution experiments.

The model presents two threshold conditions associated to the minimal $\left(B_{\min }\right)$ and maximum $\left(B_{\max }\right)$ allowed cell biomasses, which determine death and division. If $B_{r} \leq B_{\text {min }}$, the cell dies and is therefore removed from lattice, i. e. $B_{r} \rightarrow 0, S_{r} \rightarrow 0, J_{r} \rightarrow 0$. If, on the other hand, $B_{r} \geq B_{\max }$, the cell achieves its maximum allowed size and growth stops.

If the cell has achieved its maximum size and there is a neighbour empty site $S_{r^{\prime}} \in \Gamma_{r}$, the cell will divide in two identical cells, generating two new states, one in the position $S_{r}$ previously occupied by the mother cell, and one in the hitherto empty site $S_{r^{\prime}}$, with $B_{r}, B_{r^{\prime}}=B_{\max } / 2$ and $S_{r}, S_{r^{\prime}} \rightarrow 1$. As per the adhesion parameter $J$, this can be transmitted to the offspring unvaried, or mutated with a probability $P_{\mu}$. If mutation occurs, a change is introduced in the adhesion of the daughter cell in $S_{r^{\prime}}$, namely $J_{r^{\prime}}=J_{r}+\Delta J$ where $\Delta J$ is a positive or negative number randomly drawn from a normal distribution with $\sigma=\sigma_{\mu}$. By including this rule, we allow the evolution of adhesion or repulsion, starting from an initial population where $J$ is set to zero for all cells.

\section{RESULTS}

We have studied the evolutionary dynamics of adhesion starting from an initially neutral $(J=0)$ population of cells. Specifically, at time zero cells are inoculated on $25 \%$ of the sites of the circular resource area $\Psi$, located 

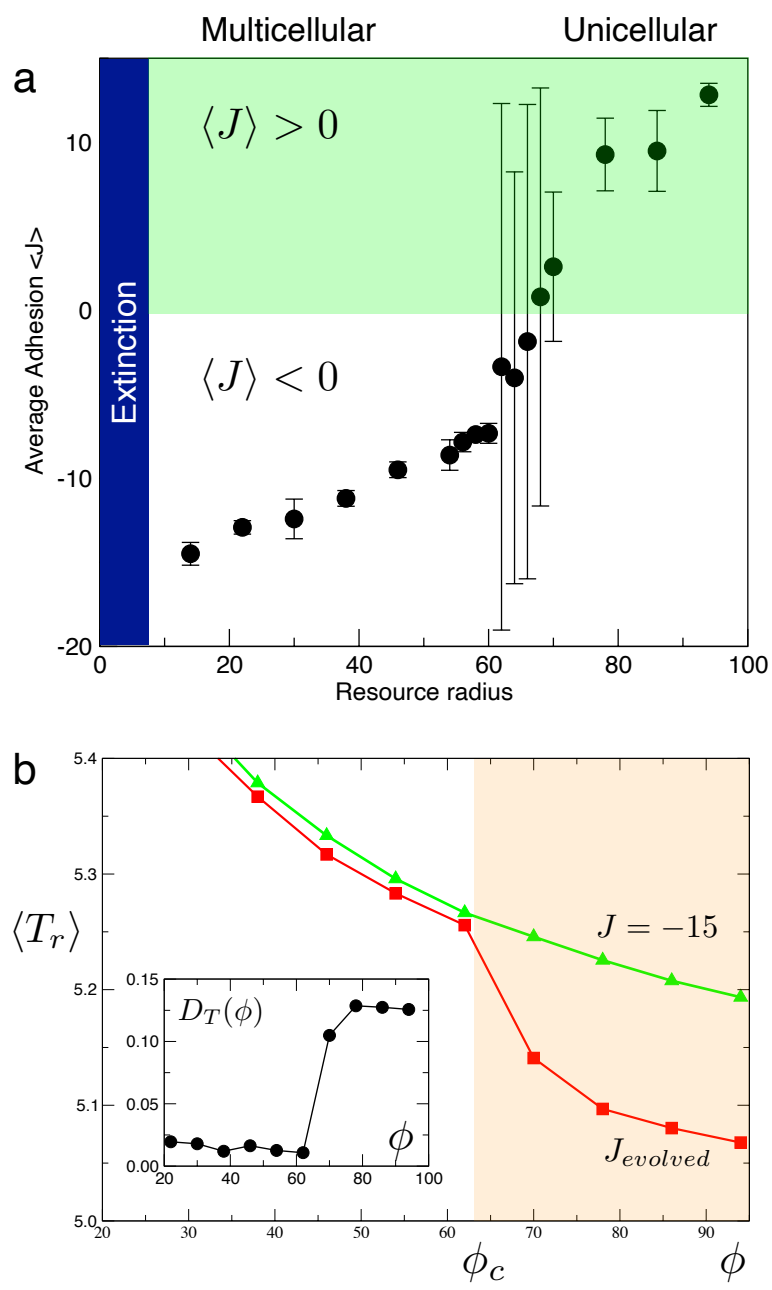

FIG. 3: (a) Transitions from multi- to unicellularity, as displayed by the values of $\langle J\rangle$ against $\phi$, by averaging over ten replicas of each evolutionary experiment. Error bars indicate the standard deviation. In (b) we show the average cell reproduction time $\left\langle T_{r}\right\rangle$ against resource size. Here the evolved values of $\langle J\rangle$ are compared with the expected results for a fixed $J=-15$ value associated with multicellular aggregates. A marked divergence occurs close to $\phi_{c}$ as unicellularity is chosen, allowing for faster replication. The difference between both curves is shown in the inset.

at the center of a $L \times L$ lattice $(L=200)$. The initial biomass for each cell is randomly set between $10^{-2}$ and 0.1 a.u. (arbitrary units of energy). The set of fixed parameters of our model is indicated in table I. In our simulations populations evolve over a maximum of $T=$ $2 \times 10^{5}$ Monte Carlo steps. Each step involves $L \times L$ asynchronous updates in which a random site is chosen and the previous rules are applied.

\section{A. Evolving adhesion under resource constraints}

By evolving individual adhesion values as described above, we test the idea that selection for adhesion is ef- fective when resources are localised in space. Nutrient is constantly introduced in the $\Psi$ area, in such a quantity that in the inner areas of the disc cells can reproduce effortlessly and death by lack of energy is extremely unlikely. On the contrary, cells that move away from the nutrient disc are not able to reproduce and rapidly die due to lack of energy. In particular, a simple calculation from equation 5 shows that under full occupation (i.e. when the normalised population $P=1$ ), the value of nutrient at equilibrium will be:

$$
R^{*}=\frac{\rho_{R}}{\eta_{R}+\xi_{R} P}=\frac{\rho_{R}}{\eta_{R}+\xi_{R}}=0.057 \text { a.u. }
$$

and since the level of biomass at which reproduction occurs is $B_{\max }=0.05$ a.u., reproduction of cells inside the nutrient disc will be effortless.

To introduce resource size as a parameter, we consider the diameter $\phi$ of the $\Psi$ domain. Four examples are shown in Fig. 2a (top). The average adhesion rate $\langle J\rangle$ is computed at each Monte Carlo step, namely:

$$
\langle J\rangle(t)=\frac{\sum_{r \in \Omega} J_{r}(t) S_{r}(t)}{\sum_{r \in \Omega} S_{r}(t)}
$$

for $t=0, \ldots, T$. In Fig. 2 we show the evolution of the average adhesion for different resource sizes. We can see that, for small sizes, $\langle J\rangle$ evolves towards negative values (self-adhesion), favouring aggregation between cells. If we increase the size of the resource, we reach a critical value $\phi_{c}$ of the diameter after which adhesion will evolve towards positive values (self-repulsion).

Close to $\phi_{c} \approx 60$, a marked transition occurs. This is summarised in Fig. 3a, where the previous runs have been averaged over 10 independent evolutionary experiments. Above a given minimum resource size, under which populations decline to extinction, two main phases can be distinguished, associated to multicellular (for $\phi<\phi_{c}$ ) or unicellular behaviour (for $\phi>\phi_{c}$ ). For small resource sizes, cells evolve self-adhesion, i.e. they implement an efficient mechanism to integrate consensus nutrient sensing and ensure that they remain close to the available source of energy. A steep increase in the values of average adhesion for $\phi \sim \phi_{c}$ indicates that the aggregative behaviour loses its efficiency over the free cell state (UC behaviour) for big resource sizes.

In each of the two phases cells carry out a different response to the surrounding density of cells. To see this, let us start with the original definition of the transition probability (4). We can see that, assuming that two neighbouring cells have been chosen, i, e, $S_{r} S_{k}=1$, we just have $\mathscr{H}=\sum_{k}\left(J_{r}+J_{k}\right) / 2$. If we consider that in each population (or sub-population, as we will see) of cells $\langle J\rangle$ displays a small variance and thus $J_{r} \sim J_{k}=\langle J\rangle$, we can write, setting $T=1$ :

$$
\begin{array}{r}
P(k \mid r) \sim \frac{1}{1+e^{\langle J\rangle\left(q\langle S\rangle^{\prime}-q\langle S\rangle\right)}} \\
\sim \frac{1}{1+e^{\langle J\rangle\left(\rho^{\prime}-\rho\right)}}
\end{array}
$$



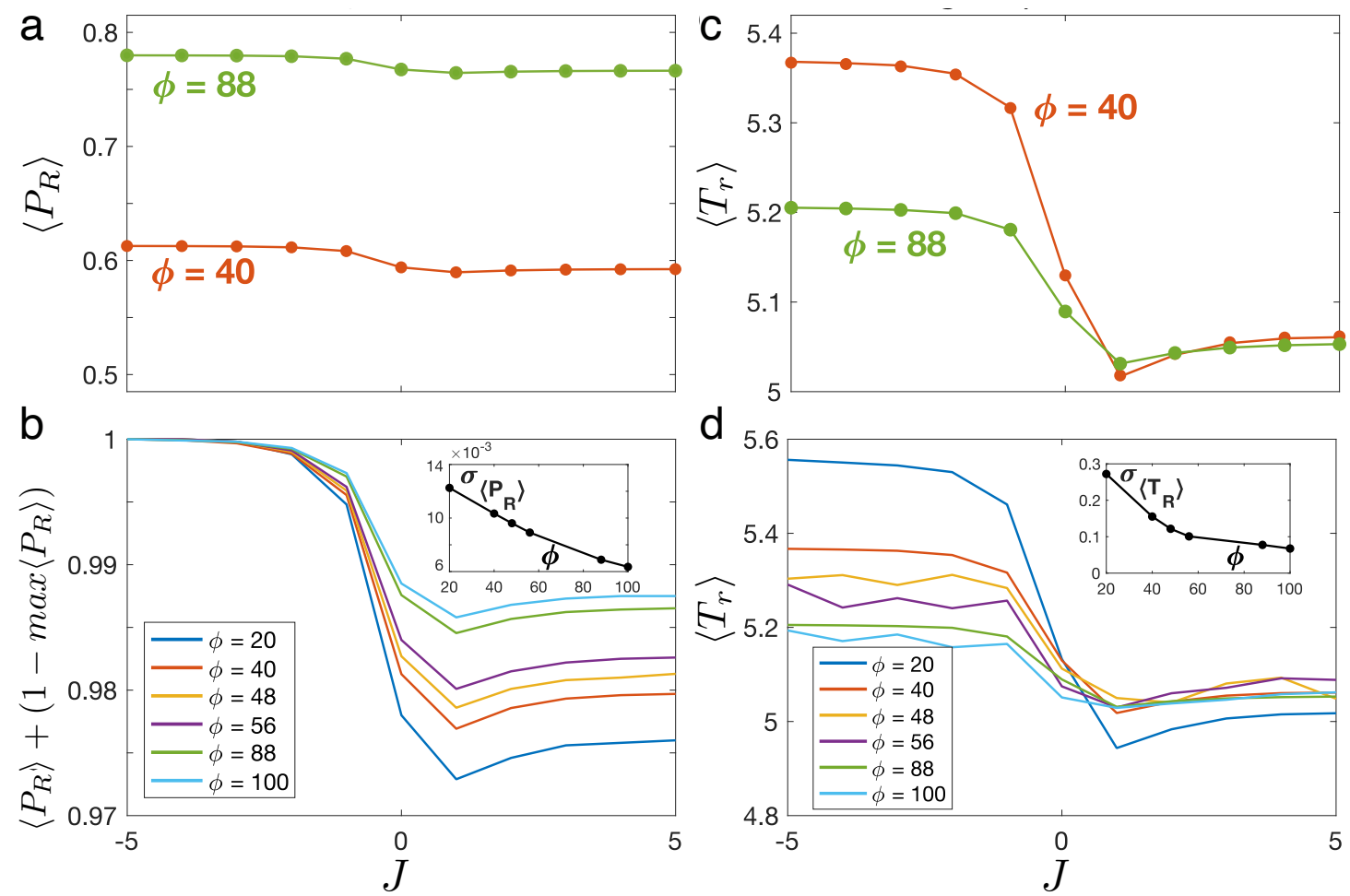

FIG. 4: (a) average fraction $\left\langle P_{R}\right\rangle$ of the population occupying $\Psi$ for fixed diameters $\phi=40\left(\phi<\phi_{c}\right)$ and $\phi=88\left(\phi>\phi_{c}\right)$, against the adhesion parameter $J$, averaged over four replicas). MC behaviour $(J<0)$ allows a better occupation of the disc. However, in the UC range $(J>0)\left\langle P_{R}\right\rangle$ decreases, with a steep transition for $J \sim 0$. Notice that the larger $\Psi$, the larger the average occupation $\forall J$. In (b) we normalise to one the maximum value of each plot using $\left\langle P_{R}\right\rangle+\left(1-\left\langle P_{R}\right\rangle\right)$. Note that the range between $\left\langle P_{R}\right\rangle_{\min }$ and $\left\langle P_{R}\right\rangle_{\max }$ decreases as $\phi$ increases, indicating that the impact of choosing MC over UC behaviour becomes less relevant as $\phi$ increases. In the inset we show the same effect, using as a measure the standard deviation $\sigma$ of the data points of each distribution with fixed $\phi$. In (c) we show the average reproduction time of cells over four replicas, for $\phi=40$ and $\phi=88$ against $J$. UC provides an advantage (faster reproduction) over MC. For larger values of $\phi$ the reproduction time decreases for $J<0$ (MC behaviour), indicating also here that the impact of choosing MC over UC behaviour becomes less relevant as the size of nutrient disc $\Psi$ increases. In (d) we show the same effect for multiple values of $\phi$. In the inset, we show that the standard deviation $\sigma$ among all datapoints of each plot with fixed $\phi$ becomes smaller as the size of the nutrient disc $\Psi$ increases.

where we define the average densities of occupied sites by $\rho=q\langle S\rangle$ for $q$ nearest neighbours ( $q=8$ in our case). For systems where densities are similar (i. e. if cells are equally scattered over $\Psi$ ) we have $\rho^{6}-\rho \ll 1$ and a Taylor expansion gives:

$$
P(k \mid r) \sim \frac{1}{2}\left(1-\frac{1}{2}\langle J\rangle\left(\rho^{\prime}-\rho\right)\right)
$$

This result indicates that, under the previous approximations, the probability of moving of a given cell located at $r \in \Omega$ is proportional to the density difference between the current and the alternative state. For positive $\langle J\rangle$, this means that movement occurs towards less dense locations. This situation reverses for negative couplings, with a probability that favours moving towards more crowded areas (i. e. adhesion).

What is being selected when resource sizes increase beyond the critical domain? Below this boundary the risk of moving away from $\Psi$ forces evolution to choose adhesion, as MC represents the right strategy to stay on top of $\Psi$ and maximise nutrient absorption. As we have shown in eq. 8, resources are always abundant on top of $\Psi$, even in case of full disc occupation, and cells can reproduce easily (because of the low reproduction threshold $B_{\max }$ defined in our model). Nevertheless, the size of the nutrient disc does in fact affect the behaviour of cells. For $\phi<\phi_{c}$, we could expect that the evolution of adhesion (i.e. of MC behaviour) is the best strategy that cells have at their disposal to ensure that they and their offspring remain on top of the nutrient disc $\Psi$. Moreover, the evolution of adhesion allows cells to effectively integrate a nutrient-sensing mechanism: two cells displaying adhesion will remain attached, reducing their mobility and thus increasing the probability that their offspring is generated on top of the nutrient disc, creating a positive feedback loop. On the other hand, as $\Psi$ becomes larger, one could expect that the probability to stay on top of the nutrient increases also for cells that are not displaying adhesion, and that therefore the need to evolve adhesion becomes less relevant. One could, therefore, explain the 
evolutionary emergence of UC over MC for $\phi>\phi_{c}$ by speculating that UC provides some other kind of advantage over MC, which for $\phi>\phi_{c}$ becomes dominant over the advantage of increased nutrient occupation that led to the selection of $\mathrm{MC}$ for $\phi<\phi_{c}$.

In Fig. 3b we show that, as $\phi$ grows, the evolutionary dynamics of adhesion result in an average reproduction time $\left\langle T_{r}\right\rangle(\phi)$ that displays a marked, discontinuous decay at $\phi \sim \phi_{c}$. This is highlighted by a simple comparison with the expected average time shown by a nonevolutionary scenario in which cells always display adhesion (upper curve, where we have used a representative fixed value $J=-15$ ). How should we explain the selection of UC over MC for $\phi>\phi_{c}$ ? We have already seen that $\mathrm{MC}$ and $\mathrm{UC}$ provide different advantages to cells (increased occupation of the nutrient disc $\Psi$ and reduced reproduction time respectively), and that the advantages of $\mathrm{MC}$ over $\mathrm{UC}$, which are relevant for a small nutrient disc, become less important as $\phi>\phi_{c}$. One possible additional explanation for the selection of UC when $\phi>\phi_{c}$ could be given by the fact that, since larger nutrient discs display a larger $\partial \Psi$ (the circumference of diameter $\phi$, i.e. the ring at the border of the nutrient patch), there is a larger boundary at the interface between nutrient and empty space where division can occur more efficiently compared with the inner areas of the disc (where there are less empty spaces available for cells to reproduce), and UC selection might help cells to better exploit this opportunity. In fact, the more pronounced slope associated to the evolved system for $\phi \sim \phi_{c}$ suggests that above $\phi_{c}$ much more opportunities for replication are allowed, perhaps due to the much larger surface of $\Psi$. This difference is highlighted in the inset of Fig. $3 \mathrm{~b}$ using a relative measure $D_{T}(\phi)=\left(T_{r}\left(J_{-15}\right)-T_{R}\left(J_{\text {evol }}\right)\right) /\left(\left(T_{r}\left(J_{-15}\right)+T_{R}\left(J_{\text {evol }}\right)\right)\right.$ where a marked transition is observed.

To find out what features of the system change in the selection of MC or UC, we have further analysed the statistical behavior of $\left\langle T_{r}\right\rangle$, as well as the fraction $\left\langle P_{R}\right\rangle$ of the population that occupies $\Psi$. In Fig. 4 we run a series of non-evolutionary simulations to plot how $\left\langle P_{R}\right\rangle$ and $\left\langle T_{r}\right\rangle$ vary in function of $J$, for different values of $\phi$. We show that the selection of MC $(J<0)$ effectively increases the average occupation $\left\langle P_{R}\right\rangle$ of the nutrient disc by cells (Figs. 4a, 4b). On the contrary, the selection of $\mathrm{UC}(J>0)$ reduces reproduction time (Figs.4c, 4d). Therefore evolving MC effectively provides cells with an advantage over nutrient occupation, while the selection of UC gives cells an advantage in terms of decreased replication time. Figure 4 also shows that the range between $\left\langle P_{R}\right\rangle_{\min }$ and $\left\langle P_{R}\right\rangle_{\max }$ (Fig. 4b) and between $\left\langle T_{R}\right\rangle_{\min }$ and $\left\langle T_{R}\right\rangle_{\max }$ (Fig. $4 \mathrm{~d}$ ) decreases as $\phi$ increases, indicating that the impact of choosing $\mathrm{MC}$ over $\mathrm{UC}$ behaviour becomes less relevant as the size of nutrient disc $\Psi$ increases.

This transition supports a scenario of evolved (simple) multicellularity where resource scarcity might have favoured cells to stick together in order to efficiently ex- ploit spatially localised nutrients. Within our special choice of the landscape, made by a single circular patch of resources, the possibility that moving around at random drives a cell to a low-nutrient area acts as a powerful selection for adhesion. In the next section we will see how this works in a spatially explicit context.

\section{B. Evolutionary branching}

The transition from multicellular to unicellular is defined by a narrow domain where very broad fluctuations are observed. This is dramatically shown by the high standard deviations associated to $\langle J\rangle$ (Fig. 3a) What is happening at these domains? The observed transition pattern does not trivially map with standard phase transitions, where the presence of a divergent increase in the variance of the patterns is a common trait (Solé, 2011). In standard transitions, the variance diverges due to temporal fluctuations: the average value of the quantity of relevance (the so-called order parameter) changes in time, moving between a broad range of possible values. That would mean, in our case, that the average adhesion would move repeatedly from positive to negative values and back. For so-called critical transitions, the given average quantity (the order parameter) just widely fluctuates (Sethna, 2006). This is not the case here.

Interestingly, and by contrast with standard phase transition phenomena, the origin of this variance has to be found somewhere else. Here, it is explained as an evolutionary branching process (EBP) where different values of $J$ evolve from the original zero- $J$ state. In EBP, frequency-dependent selection splits into two (or more) distinct phenotypic clusters (Geritz et al., 1997; Doebeli and Dieckmann, 2000). In Fig. 5, we display a set of branching patterns obtained by plotting the $J$ value of each cell present over the evolutionary experiment. For values below $\phi_{c}$ only one branch is obtained (Fig. 5a), as the entire population of cells evolve individual adhesion towards negative values ( $M C$-only phase) with a low standard deviation associated to $\langle J\rangle$. For values above $\phi_{c}$, on the other hand, sub-populations emerge: the majority of cells evolve their adhesion toward positive values (UC), but a minor portion of cells bifurcates from the main branch, evolving a MC sub-population ( $U C$ - $M C$ phase). This lesser MC sub-population emerges mainly on the external border of the nutrient disc, at the interface between nutrient and empty medium, and is eventually superseded by the prevailing UC sub-population. As soon as the MC branch is permanently extinguished, a new $\mathrm{MC}$ branch diverges from the main UC branch (Fig. 5c). As the majority of cells is part of the UC population, also for $\phi>\phi_{c}$ the standard deviation associated to $\langle J\rangle$ is low. Interestingly, branching and subsequent extinction of these MC branches occur at a higher pace as $\phi$ increases. Finally, for $\phi \sim \phi_{c}$ the two phases will coexist, with some simulations in the transition zone leading to the UC-MC phase (Fig. 5b), and some others 

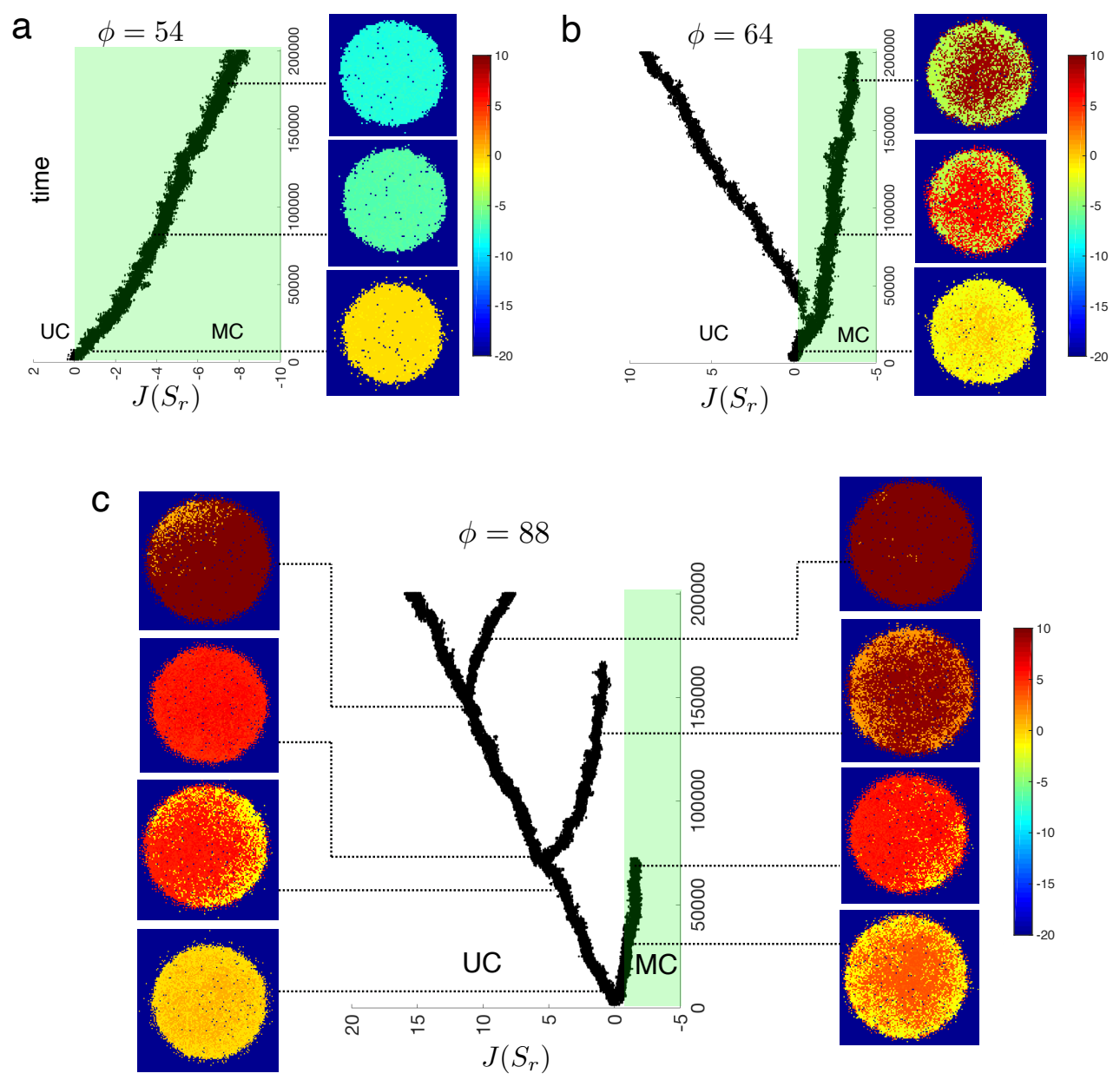

FIG. 5: Evolutionary branching under resource constraints. Three different examples of the evolutionary patterns displayed by the evolved adhesion model for three different resource sizes. For sub-critical $\phi$, only one branch is found (a) associated to the emergence of multicellular ensembles. For super-critical $\phi$ (c) multiple branches are found, with cells on the periphery $\partial \Psi$ evolving adhesion while those within $\phi$ display repulsion. Here an arms race between the two kinds of phenotypes can be seen. In the critical domain where $\phi \sim \phi_{c}$ the system can evolve indifferently towards the UC-MC phase that is typical of super-critical $\phi(b)$ or towards the MC-only phase that is typical of sub-critical $\phi$ (not shown).

leading instead to the MC-only phase (not shown). The coexistence of the two phases explains the high standard deviation associated to $\langle J\rangle$ in the in the $\phi \sim \phi_{c}$ area.

We have seen that in the UC-MC phase no longterm coexistence is found between the UC and the MC branches. Instead, extinction events cyclically occur as low-adhesion subpopulations emerge and eventually decline. What is the origin of the pattern? In our case study, where asexual populations are considered, the origin of this EBP must be found in an intrinsic heterogeneity associated with the resource spatial arrangement. Here our domain $\Psi$ defines a closed surface delimited by its boundary $\partial \Psi$ (the circumference of diameter $\phi$ ). Because of the sharpness of the boundary condition, cells living within or close to the border of $\Psi$ will experience markedly different constraints. While the former will mainly compete with neighbouring ones for available resources, those close to the boundary face a strong selection: if their $J$ values are high, they will more easily get expelled outside the safe zone. What is then the spatial patterning associated to our evolutionary model? Seminal work on evolution of asexual populations experiencing environmental gradients (Doebeli and Dickmann, 2003) revealed that speciation is likely to occur. Our system is an extreme case of such a spatially heterogeneous scenario.

In order to visualise what occurs at the microscopic level, we can follow the time evolution of the spatial arrangement of cells in terms of their adhesion values. These snapshots are shown in Figs. 5a-c) along with their time location. For the small resource case (Fig. 5a) all cells have a very similar, negative $J$ values and the spatial patterns are homogeneous. For the two-branch case (Fig. 5b) three plots of the spatial state are displayed, revealing the origin of the two different sets of phenotypes.

As we pointed out before, a distinct population of cells displaying adhesion emerges at the boundaries of the re- 

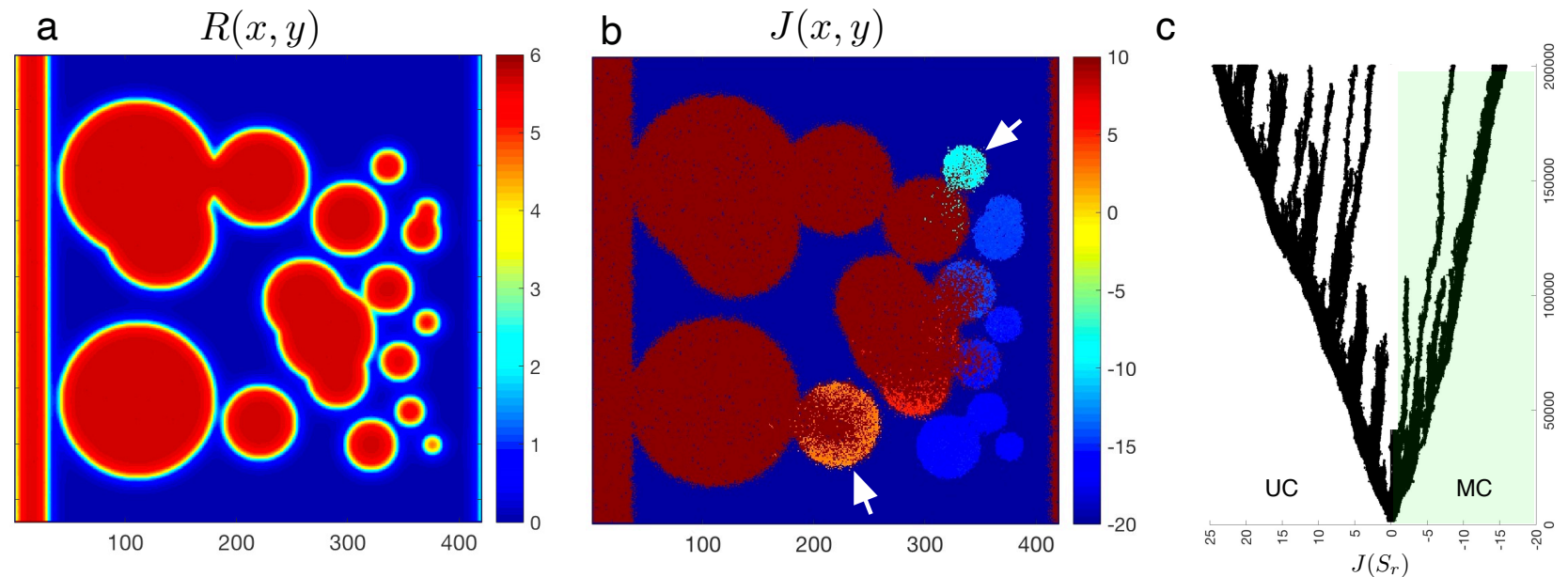

FIG. 6: Evolutionary branching in a heterogeneous resource landscape. Using a complex spatial distribution of patches (a) including both continuous (left wall), large and small patches, following a decreasing pattern, evolved populations display a whole range of adhesion values (b). The arrows indicate stable resource patches that display adhesion and persist in a stable manner despite their connection with larger patches dominated by repulsion. The evolution of diversity can be visualized as a multibranched tree (c).

source, achieving a stable population size. This stable branching changes when larger resource sizes are considered, as shown in the snapshots of Fig. 5c. Here the same previous pattern is observed at the beginning of the in silico evolution experiment $(t<6500)$. At this point, the cell population on $\partial \Psi$ decreases and gets extinct. A new homogeneous state emerges that then evolves again into a heterogeneous population where lowadhesion rates evolve (evolving towards lower values of $J$ i.e. towards adhesion, although not reaching negative values) until a new extinction (and in turn a new branching) occurs. In other words, a combination between evolutionary branching and Red Queen dynamics unfolds.

The previous examples involve a very simple, geometric description of the resource landscape. What is the impact of considering a rich, heterogeneous distribution of resources? An example of this scenario is displayed in Fig. 6. Here we have created a landscape of resource patches that follows a left-right gradient of decreasing size, with a (infinite radius) continuous band on the left wall to small circles on the right (fig. 6a). The evolutionary experiment is run again under the same conditions as above and in this case large and small patches can interact through spatial interconnection (either directly or through cell migration, fig. $6 \mathrm{~b}$ ). The result from this in silico evolutionary experiment is a multi-branching pattern (fig. 6c) where new branches and extinctions occur with a long-term coexistence of multiple phenotypes (as defined by our single parameter space).

A relevant result here concerns the impact of spatial embodiment as a firewall against invasion. As shown in Fig. 6b, large and small patches can exchange cells and thus invasion by cells from one large domain could in principle overcome the population evolved in the smaller one, but this is often not the case. Fig. $6 \mathrm{~b}$ provides a clear example: Here two smaller domains in contact with larger ones dominated by repulsion keep their adhesive nature over evolutionary time in a stable manner. Because of the selective pressure exerted by the boundary, both types of $J$ values coexist in time.

All the results shown in this section have been obtained in a evolutionary model where the adhesion parameter $J$ can vary freely both towards adhesion $J<0$ and repulsion $J>0$, with cells actively avoiding each other in the repulsion zone. We also tested all the obtained results in a more relaxed model, where only evolution in the range $(J \leq 0)$ is allowed, i.e. where active repulsion cannot be selected: at $J=0$ cells simply move randomly. All the results shown above were also obtained, indicating that our results are robust.

\section{DISCUSSION}

In this paper we have presented a minimal model of embodied evolution of multicellularity resulting from a selection pressure tied to resource abundance. The model is limited to the possible paths leading to clumps of cells that get together without defining a true multicellular organism. Clumping has been previously explored using mathematical models that consider multicellular clumps as 'public goods' (Biernaskie and West, 2015) that could facilitate the emergence of MC traits. Here the clumps are explicitly defined as embodied systems and we refer to them as simple multicellularity, for which efficient exploitation of patchy resources is a main driver (Tong et al. 2021). Specifically, we have used a simple resource description as a circular area $\Psi$ whose linear size (the diameter $\phi$ ) is used as a key parameter. By increasing resource 
size, its relative impact on cell-cell interactions is measured by means of a single, evolvable parameter, namely the adhesion coupling $J$, which starts from zero and can evolve to either positive or negative values. These correspond to either repulsion (cells tend to avoid each other) or adhesion (cell-cell attachment), respectively.

The model is a physics-based approach, where an energy function can be easily defined that allows defining probabilistic rules of cell-cell exchanges. Following the standard view of physical interactions between components in a strongly interacting system, changes occur in the direction of minimising energy. This kind of model provides the proper framework to simulate the spatial dynamics of strongly interacting systems (Goldenfeld, 2018) incorporating collective cell behaviour (Graner and Glazier, 1992). Additional rules include also the intake and metabolic burden of cells, along with reproduction and death that take place once given biomass thresholds are reached.

The evolution of adhesion occurs due to the strong selection imposed by limited available local resources. When the size of the resource is small, the risk of reaching the boundary $\partial \Psi$ and getting out of the nutrient source makes adhesion to other cells within $\Psi$ a desirable trait. Cohesive groups can thus allow their members to remain alive, although their potential for replication is reduced due to the limited space available. Instead, if resource limitations are reduced due to the large size of $\Psi$, a cell within the patch might easily replicate and find resources in the neighbourhood also in absence of strong adhesive traits. The exception is of course given by those locations close to the boundaries: in that area movement can drive cells outside of the safety of $\Psi$ and there is thus an increased pressure for avoiding movement. As it occurs with previous models of speciation of asexual populations in heterogeneous landscapes, heterogeneity is a driver of diversity, which allows the coexistence of both MC and UC for large $\Psi$ (i.e. when $\phi>\phi_{c}$ ). Our model provides a new illustration of this phenomenon under an embodied, physical picture.

Our results have been obtained by means of the simplest formal approximation. We have not taken into account relevant features of cells, such as shape, that can be captured by extended models of spin interactions where each cell is defined not by a single unit but multiple, interconnected sites (Graner and Glazier, J. A.1992; Glazier and Graner, 1993; Savill et al. 1997). We have also limited the internal description of cells to a basic set of variables that encapsulate metabolism and cell cycle ignoring in particular the potential role played by diffusion across cell-cell channels as a trigger for MC cooperation (Knoll, 2011). Future work should consider all these possible refinements of our work, as well as the introduction of additional features, such as the presence of genetic control mechanisms that could allow the evolution of additional relevant properties, including life cycles, responses to stress or interactions with a diverse range of (negative and positive) external clues and cell differentiation (Hogeweg, 2000; Márquez-Zacarias et al, 2021). Similarly, our description lacks any gene network description where different functionalities beyond adhesion could be included and would allow modelling the problem from the unicellular perspective (Sebe-Pedros et al., 2017; 2018). Finally, the simple approach taken here can inspire further work involving synthetic biology, where engineering multicellular traits have been a major research target (Chuang, 2012; Solé et al., 2018) and can provide insight into its early evolutionary origins (Solé, 2016).

\section{ACKNOWLEDGMENTS}

The authors thank the Complex Systems Lab members for fruitful discussions. This work has been supported by the Spanish Ministry of Economy and Competitiveness, grant PID2019-111680GB-I00, an AGAUR FI 2018 grant, and the Santa Fe Institute.

\section{References}

1. Biernaskie, J.M. and West, S.A., 2015. Cooperation, clumping and the evolution of multicellularity. Proc. R. Soc. B 282, 20151075.

2. Bonner, J. T. 2001. First signals: the evolution of multicellular development. Princeton U. Press. Princeton.

3. Chuang, J. S. 2012. Engineering multicellular traits in synthetic microbial populations. Curr. Opin. Chem. Biol. 16, 370-378.

4. Deutsch, A. and Dormann, S., 2005. Mathematical modeling of biological pattern formation. Birkh" auser, Boston.

5. Doebeli, M. and Dieckmann, U., 2000. Evolutionary branching and sympatric speciation caused by different types of ecological interactions. The american naturalist, 156, S77-S101.

6. Duran-Nebreda, S., Bonforti, A., Montanez, R., Valverde, S. and Solé, R., 2016. Emergence of proto-organisms from bistable stochastic differentiation and adhesion. J. Roy. Soc. Interface 13, 20160108.

7. Forgacs, G., and Newman, S. A. 2005. Biological physics of the developing embryo. Cambridge $\mathrm{U}$. Press, Cambridge.

8. Foty, R. A. and Steinberg, M. S. 2005. The differential adhesion hypothesis: a direct evaluation. Developmental biology 278, 255-263.

9. Glazier, J. A. and Graner, F. 1993. Simulation of the differential adhesion driven rearrangement of biological cells. Phys. Rev. E 47, 2128-2154. 
10. Goldenfeld, N., 2018. Lectures on phase transitions and the renormalization group. CRC Press.

11. Graner, F. and Glazier, J. A. 1992. Simulation of biological cell sorting using a two-dimensional extended Potts model. Phys. Rev. Lett. 69, 20132016.

12. Geritz, S.A., Metz, J.A., Kisdi, E. and Meszena, G., 1997. Dynamics of adaptation and evolutionary branching. Phys. Rev. Lett. 78, 2024.

13. Hogeweg, P., 2000. Evolving mechanisms of morphogenesis: on the interplay between differential adhesion and cell differentiation. J. Theor. Biol. 317-333.

14. Knoll, A.H., 2011. The multiple origins of complex multicellularity. Annu. Rev. Earth Planet. Sci. 39, 217-239.

15. Libby, E. and Ratcliff W. C. 2014. Ratcheting the evolution of multicellularity. Science 346, 426-427

16. Maharbitz, M. M. 2012. Synthetic multicelullarity. Trends Cell Biol. 22, 617-623

17. Márquez-Zacarías, P., Pineau, R.M., Gomez, M., Veliz-Cuba, A., Murrugarra, D., Ratcliff, W.C. and Niklas, K.J., 2021. Evolution of cellular differentiation: from hypotheses to models. Trends Ecol. Evol. 36, 49-60.

18. Newman, S. and Comper, W. D. 1990. 'Generic' physical mechanisms of morphogenesis and pattern formation. Development 110, 1-18.

19. Newman, S. A. and Baht, R. 2008. Dynamical patterning modules: physico-genetic determinants of morphological development and evolution. Phys. Biol. 5, 015008.

20. Newman, S.A., Forgacs, G. and Müller, G. B. 2006. Before programs: the physical origination of multicellular forms. Int. J. Dev. Biol. 50, 289-299.

21. Pentz, J.T., Marquez-Zacarias, P., Bozdag, G.O., et al. 2020. Ecological advantages and evolutionary limitations of aggregative multicellular development. Curr. Biol. 30, 4155-4164.

22. Queller, D. C. 2000. Relatedness and the fraternal major transitions. Phil Trans Roy Soc Lond B, 355:1647-1655.
23. Ratcliff, W. C., Denison R. F., Borrello M. and Travisano M. 2012. Experimental evolution of multicellularity. Proc. Natl. Acad. Sci USA. 109, 1595-1600.

24. Ruiz-Trillo, I. and Nedelcu, A.M. eds., 2015. Evolutionary transitions to multicellular life: principles and mechanisms (Vol. 2). Springer.

25. Savill, N.J. and Hogeweg, P., 1997. Modelling morphogenesis: from single cells to crawling slugs. J. Theor. Biol. 184, 229-235.

26. Sebé-Pedros, A., Degnan, B.M. and Ruiz-Trillo, I., 2017. The origin of Metazoa: a unicellular perspective. Nature Rev. Genetics, 18, 498-512.

27. Sebé-Pedros, A., Chomsky, E., Pang, K., LaraAstiaso, D., Gaiti, F., Mukamel, Z., Amit, I., Hejnol, A., Degnan, B.M. and Tanay, A., 2018. Early metazoan cell type diversity and the evolution of multicellular gene regulation. Nature Evol. Evol. 2, 1176-1188.

28. Solé, R., 2011. Phase transitions. Princeton U. Press. Princeton, NJ.

29. Solé, R., 2016. Synthetic transitions: towards a new synthesis. Phil. Trans. R. Soc. B 371, 20150438.

30. Solé, R., Ollé-Vila, A., Vidiella, B., DuranNebreda, S. and Conde-Pueyo, N., 2018. The road to synthetic multicellularity. Curr. Op. Syst. Biol. 7, 60-67.

31. Steinberg, M. S. 1975. Adhesion-guided multicellular assembly: a commentary upon the postulates, real and imagined, of the differential adhesion hypothesis, with special attention to computer simulations of cell sorting. J. Theor. Biol. 55, 431-432.

32. Tarnita, C.E., Taubes, C.H. and Nowak, M.A., 2013. Evolutionary construction by staying together and coming together. J. Theor. Biol. 320, $10-22$.

33. Tong, K.; Bozdag, G.O.; Ratcliff, W.C. 2021. Selective Drivers of Simple Multicellularity. Preprints 2021, 2021110194 (doi: 10.20944/preprints202111.0194.v1). 